\title{
E1 estudio de las Relaciones Internacionales a cien años de su creación como disciplina científica
}

\author{
The study of International Relations one hundred years after its \\ creation as a scientific discipline
}

Victor F. Toledo ${ }^{1}$

Citar: Toledo, V. F. (2020). El estudio de las Relaciones Internacionales a cien años de su creación como disciplina científica. Cuadernos Universitarios, 13, pp. 99-108.

Recibido: setiembre 2020

Aceptado: octubre 2020

Ensayo científico

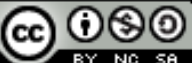

\section{Resumen}

El presente ensayo tiene como finalidad hacer un repaso del estudio formal de las Relaciones Internacionales como disciplina científica desde su emergencia — hace más de una centuria— intentando reflejar cómo los debates en torno a su objeto, metodología y opciones teóricas han incidido en la estructuración de su currícula, generándose así una situación paradójica; pues esto, a la vez que avivó su estudio y reflexión crítica fue, en cierto punto, un obstáculo para su consolidación. Como toda ciencia social, las Relaciones Internacionales están histórica y geográficamente situadas, es por ello que los contextos no pueden eludirse a la hora de realizar un abordaje de esta problemática. Como el contexto internacional es altamente dinámico y cambiante, se resalta la necesidad de acompañar esa particularidad tanto administrativa, pedagógica y didácticamente.

Palabras clave: disciplina - objeto de estudio - relaciones internacionales - transdisciplinariedad

\footnotetext{
1 Universidad Católica de Salta. Licenciado y Profesor Universitario en Relaciones Internacionales, egresado de la Universidad Católica de Salta (UCASAL). Magister en Administración de Negocios por la UCASAL. Maestrando en Ciencia, Tecnología y Sociedad (Universidad Virtual de Quilmes). Docente en las facultades de Ciencias Jurídicas, Economía y Administración y Artes y Ciencias de la UCASAL. Miembro del Instituto de Derecho Internacional de la UCASAL, del Instituto de Relaciones Internacionales y Ciencia Política de la UCASAL y de la Asociación Argentina de Derecho Internacional. Investigador Independiente «B» de la UCASAL.

vtoledo@ucasal.edu.ar
} 


\begin{abstract}
The present essay aims to review the formal study of International Relations as a scientific discipline from its emergency — more than a century agotrying to reflect how the debates around its object, methodology and theoretical options have influenced the structuring of its curriculum, thus generating a paradoxical situation; since this, at the same time that it revived its study and critical reflection was, at a certain point, an obstacle for its consolidation.
\end{abstract}

\section{Surgimiento de la disciplina}

En diferentes textos de estudio de las Relaciones Internacionales ${ }^{2}$, es común encontrar referencias a la afirmación de que se trata de una disciplina novel en el campo de las ciencias sociales, aunque su existencia como teoría sea bastante antigua. Eso es cierto, aunque en 2019 haya cumplido una centuria. El comienzo y posterior desarrollo de las Relaciones Internacionales como disciplina autónoma, que realiza un estudio de manera sistemática y que posee un objeto de estudio propio y métodos para abordarlo, tiene su acta de nacimiento con la creación de la cátedra Woodrow Wilson en Relaciones Internacionales en la Universidad de Gales (en Aberystwyth). En rigor, tenemos que aclarar que ya en el año 1900 había aparecido en Estados Unidos, por primera vez, una obra de Relaciones Internacionales en sentido propio, se trataba de Política mundial a fines del siglo XIX, escrita por Paul Reinsch. Más tarde, en 1916, en Londres, se publicó una obra colectiva denominada Introducción al estudio de las Relaciones Internacionales (Del Arenal, 2000, pp. 62-63), con lo cual puede asegurarse que, pese a los obstáculos, el cami-
Like all social sciences, International Relations are historically and geographically situated, which is why contexts cannot be avoided when addressing this problem. As the international context is highly dynamic and changing, it is very important to accompany this particularity both administratively and pedagogically, and also didactically.

Key words: discipline - object of study international relations - transdisciplinarity

no hacia las Relaciones Internacionales como disciplina científica auténtica ya estaba trazado. El Carnegie Endowment for International Peace (Fondo Carnegie para la Paz Internacional), en 1910, y el World Peace Forum (Foro por la Paz Mundial), imbuidos del pensamiento pacifista de principios de siglo, fueron las primeras asociaciones que impulsaron el surgimiento de las Relaciones Internacionales (Barbé, 1995, pp. 29-30).

En 1919 apreció la primera cátedra en la materia, que, como ya se dijo, fue el punto de arranque formal, más allá que algunos autores prefieran situarlo en 1922, cuando, también en la Universidad de Gales, se creó la primera cátedra con carácter permanente. $\mathrm{Al}$ año siguiente, en la London School of Economics, se estableció la cátedra denominada La Naturaleza de las Relaciones Internacionales.

Como puede verse, el contexto para que ello sucediese era propicio, pues Europa salía de una guerra atroz con proyección internacional, por primera vez en la historia se estaba asistiendo a la creación de una sociedad de naciones con pretensiones de establecer un mecanismo de seguridad colectiva, y Estados Unidos se constituía como un actor de primer

${ }^{2}$ En este artículo, el término Relaciones Internacionales figura en mayúsculas para referirse a la disciplina y en minúsculas para referirse a la actividad. 
orden al dejar de lado su doctrina de espléndido aislamiento ${ }^{3}$. Era lógico, entonces, que las primeras indagaciones que se hicieran sobre las Relaciones Internacionales estuviesen ligadas a las cuestiones de la guerra y de la paz. Por supuesto, se tuvo que transitar un largo camino para la conformación de las Relaciones Internacionales como un campo de estudio autónomo. La Historia, el Derecho Internacional, la Diplomacia, la Ciencia Política, la Sociología y la Economía, en la medida en que se ocuparon de las cuestiones relacionadas con las que estudia la disciplina, habían trazado ese camino. Hasta los años 90 del siglo XX se podía decir que la autonomía de las Relaciones Internacionales estaba cuestionada y era terreno común encontrar, en congresos o simposios, alguna mesa temática o alguna ponencia sobre la «autonomía» de las Relaciones Internacionales. Afortunadamente esto ya no sucede, aunque no quiere decir que haya desaparecido completamente del espectro académico.

Sin embargo, el objeto de este artículo no es focalizar en los antecedentes remotos ni en los más inmediatos de esta disciplina; sino analizar, desde 1919 hasta aquí, qué ha pasado con ella y cómo se han transmitido sus conocimientos.

Sabemos que como tal estudia al sistema internacional, o a la sociedad internacional o a la comunidad internacional —depende del enfoque de cada autor-y que ese objeto de estudio es altamente dinámico. Se han escrito innumerables páginas sobre del dinamismo y la imprevisibilidad de esta disciplina. Ahora bien, el modo de estudiarla, su contenido, ¿ha evolucionado de la misma manera?

En una disciplina dentro de la cual sus estudiosos no coinciden acerca de su objeto de estudio (sociedad, comunidad o sistema internacional ${ }^{4}$ ), tampoco en su metodología (sincrónica o diacrónica; con datos duros o blandos, o enfocada en fenómenos de micro o macronivel) ni en su para qué (comprender lo local en el marco internacional o comprender lo internacional a partir de particularidades locales). Todo ello demuestra, por un lado, su riqueza, y por otro pone de manifiesto la necesidad de contar con un abordaje más sistematizado y que cuente con una currícula acorde con el dinamismo de la disciplina.

\section{Disciplina a contracorriente}

Una de las particularidades fundamentales de las Relaciones Internacionales ha sido la de remar en contra de la tendencia académica de sus pares de las ciencias sociales. E1 imperativo científico-disciplinar del positivismo del siglo XIX impregnó fuertemente todas las áreas de estudio y junto con él la exigencia de especialización de las ciencias. El ideal renacentista del genio universal que sabía «de todo» quedó superado por la Revolución Industrial y los nuevos descubrimientos en los diferentes campos del saber. Se hacía imposible que una persona, que un científico, desde una sola disciplina, conociera acerca de todo. $\mathrm{Y}$ la misma mirada, o el mismo criterio, se aplicó a los saberes hacia dentro de una disciplina. El médico tenía que especializarse, el abogado tenía que hacerlo, el biólogo también. Había que ser especialistas en algo. En ese contexto, bajo ese imperativo, emergieron las Relaciones Internacionales como una disciplina que estudiaba un objeto de estudio particular desde diferentes ópticas. El especialista en Relaciones

\footnotetext{
${ }^{3}$ Doctrina que establecía la no intervención estadounidense en los conflictos externos en general y europeos, en particular.

${ }^{4}$ Sobre el debate en cuanto al objeto de estudio de la disciplina, véase Toledo, V. F., Introducción al estudio de las relaciones internacionales, Salta: EUCASA, pp. 32-42.
} 
Internacionales tenía que tener conocimientos de Ciencia Política, de Derecho, de Economía, de Sociología, etc. Tenía que ser, pues, un «todista». Por supuesto que abrirse paso desde esa postura no le ha sido sencillo, pues de allí arrancan las críticas a los egresados de esta carrera como profesionales que abarcan mucho pero profundizan poco. Es por esto que cobran fuerza algunas propuestas u opciones de abordar lo internacional desde otras disciplinas (la Historia, el Derecho, la Ciencia Política, entre otras). Esta lógica conduce a afirmar que si todos los fenómenos actuales son internacionales, si todo es internacional, todos están capacitados para estudiar la realidad internacional y pueden ser, por lo tanto, internacionalistas.

E1 término «internacionalista» merece un párrafo aparte. Como tal, resulta interesante, es agradable al oído, pero también altamente impreciso. Como adjetivo, dice la Real Academia Española, se refiere a un «partidario del internacionalismo», o sea, a una persona «versada en el derecho internacional» $[$ sic $]$. Si profundizamos nuestra búsqueda y nos preguntamos qué es el internacionalismo, encontraremos que es definido como «(...) una doctrina o actitud que antepone lo internacional a lo nacional o una ideología - la socialista- que preconiza la asociación internacional de los obreros para obtener ciertas reivindicaciones». En definitiva, desde esta perspectiva, quien se asuma como internacionalista, a) se reconocería como versado/a en el Derecho Internacional, b) tendría que abrazar el ideario socialista, y c) quedarían al margen quienes estudian lo internacional desde la perspectiva o desde la formación en nacionalismo, o desde su defensa. En épocas en la que la autopercepción parece imponerse, quizás la discusión sea menor, pero parece más acertada la denominación tradicional —y quizás conservadora- de licenciado en Relaciones Internacionales.
En este punto se hace necesario reflexionar sobre el planteo de la enseñanza de la disciplina en cuanto tal. La necesidad de estructurar planes de estudio y contenidos específicos que indaguen el objeto de estudio desde la mirada de las Relaciones Internacionales, a su vez con el apoyo de otras disciplinas, requiere la articulación vertical y horizontal de la caja curricular, sus contenidos mínimos y los objetivos propuestos, teniendo en la mira las competencias con las que se quiere formar a los futuros egresados.

Una segunda cuestión que marca un nacimiento a contracorriente de la disciplina es la metodología. El positivismo ha dominado el espectro de la exigencia metodológica en el siglo XIX y claramente durante la primera mitad del siglo XX. Por consiguiente, se insistió en la necesidad de la explicación, de la comprobación cuantitativa, de la causalidad, de la formulación de leyes. En ese marco dominante, los estudios en relaciones internacionales se edificaron con base en una corriente interpretativa, crítica, que daba más importancia a la comprensión que a la explicación, y en cuyo seno la cuantificación carecía de sentido. Cuando en el campo general de las ciencias sociales empezó a abrirse y a ganar peso la corriente interpretativa y constructivista, a partir de la Segunda Guerra Mundial, en las Relaciones Internacionales irrumpieron con fuerza los estudios cuantitativos-explicativos, dando origen al segundo debate, es decir, el metodológico. Enfrascados en una auténtica batalla entre enfoques tradicionalistas, que afirman que únicamente se puede estudiar a las Relaciones Internacionales apelando a la historia e interpretándola en función de las necesidades y realidades actuales, y los cientificistas, que sostienen que solo hay avances en tanto y en cuanto se puedan generar modelos abstractos que midan y sustenten empíricamente cual- 
quier afirmación, los estudiosos de las relaciones internacionales demoraron su consolidación como disciplina. El segundo debate en Relaciones Internacionales, enmarcado en los años 50 y 60 del siglo $X X$, no fue únicamente formal, pues la observación del objeto de estudio está enraizada en el cómo se lo observará. Puede afirmarse que, con el paso del tiempo, existe una menor polarización entre ambas perspectivas, pero claramente no hay una síntesis o unificación entre ambas (Dougherty y Platzgraff, 1993, p. 39).

Este segundo debate no debería ser considerado como meramente formalista sino que debería ser tenido muy en cuenta a la hora de la enseñanza de una metodología de la investigación acorde con las particularidades del objeto y de lo que se necesita abordar de ese objeto en esta disciplina. Sería impreciso identificar lo cualitativo con el enfoque tradicionalista y lo cuantitativo con el enfoque cientificista, pues este último tiene como eje, en rigor, al behaviorismo (o teoría del comportamiento) (Del Arenal, 2000, p. 114). En cambio, resulta atinado tomar sus reflexiones, críticas y propuestas como mecanismos que necesitan complementarse en aras de llegar a descubrimientos y afirmaciones relevantes y respaldadas con datos.

\section{Los énfasis}

El desarrollo de una disciplina no está aislado del contexto en el que se desenvuelve. Los cien años de esta disciplina lo demuestran claramente y los énfasis que se han realizado en cuanto al estudio del objeto de la disciplina también.

La Economía es un claro ejemplo de ello. Como una actividad científica puede decirse que se origina en la Investigación sobre la naturaleza y causas de la riqueza de las naciones, obra de Adam Smith. Lo que Smith planteaba es que las políticas mercantilistas que se aplicaban, donde el interés estaba centrado en acumular oro, exportar lo más posible y cerrar las puertas a las importaciones, no generaban beneficios. Proponía que las naciones se beneficiarían mutuamente con el libre comercio, en virtud del cual cada una aprovecharía sus ventajas comparativas, abriendo el camino hacia el liberalismo, junto con figuras como las de David Ricardo y John Stuart Mill. Estas ideas fueron precursoras de las teorías liberales en Relaciones Internacionales, en la medida en que entendían que el comercio internacional favorecería a la paz (Waltz, 1959, pp. 129-130). También el marxismo hizo de la Economía un eje central en las Relaciones Internacionales. Distintas teorías se nutrieron de sus ideas para estudiar las relaciones internacionales. Las teorías imperialistas de Rosa Luxemburgo, la de Lenin, la teoría de la dependencia, la del sistema mundo, los estudios sobre el desarrollo y subdesarrollo, entre otras. En realidad, todas las teorías contenidas en el paradigma estructuralista ponen el acento en cuestiones económicas para entender el objeto de estudio postulado (Barbé, 1995, p. 69). No es casual que la primera obra contemporánea sobre relaciones internacionales, abordada desde la perspectiva de las Relaciones Internacionales, sea el libro de Carr, The Twenty Years' Crisis, que analiza las relaciones internacionales en el contexto de la crisis de 1929-1930. En dicha obra, el autor afirma que «el pensamiento político es en sí mismo una forma de acción política. La ciencia política no solo es la ciencia de lo que es, sino también de lo que debería ser ${ }^{5}$ (Carr, 2004, p. 37).

\footnotetext{
${ }^{5}$ Carr denomina a la disciplina como Ciencia de la Política Internacional.
} 
Ahora bien, las Relaciones Internacionales adquirieron la categoría de disciplina científica en el transcurso de la Guerra Fría, ante todo gracias a los aportes del realismo y neorrealismo. De manera tal que las Relaciones Internacionales estuvieron empapadas de las categorías conceptuales y de las preocupaciones de este período histórico, es decir, por la guerra, la seguridad y la carrera armamentista. En ese marco, la Economía pasó a otro plano en el estudio y análisis. A medida que avanzaba el siglo XX, los Estados dejaron de ser considerados como los únicos actores internacionales, y las organizaciones multinacionales y las corporaciones transnacionales adquirieron mayor peso en la toma de decisiones internacionales. Así, mientras que el realismo clásico dejó de lado a la Economía a la hora de teorizar sobre las Relaciones Internacionales, el neorrealismo tuvo que darle cabida en su complejo teórico. Es más, para hacerle frente emergió el paradigma trasnacionalista.

A fines de la década del 70 del siglo XX, con la crisis energética que sacudió a todo el mundo, se introdujo la noción de Economía Política Internacional (EPI), impulsada por Susan Strange ${ }^{6}$. Tras la finalización de la Guerra Fría y la aceleración de la globalización, se observa cómo varias corrientes teóricas hacen pie en cuestiones económicas (Toledo, 2006, p. 54), lo que impactó fuertemente en los planes de estudio y carreras de posgrado.

Así como la referencia a la Economía es innegable, también lo es a la Ciencia Política, disciplina que por mucho tiempo acaparó a las Relaciones Internacionales en su égida. El estudio del poder y la utilización de la terminología Política Internacional es muestra de ello. En ese sentido, el realismo clásico ha sido fundamen- tal, particularmente al trazar la clara distinción entre la política interna y la internacional, como disciplinas que van por carriles diferentes y que no obedecen a la misma lógica. Una vez más, la aceleración de la globalización ha cambiado esta concepción. La glocalización, palabra con la que Ullrich Beck alude a la coexistencia, interrelación y conflicto entre lo global y lo local (1998, p. 77) obliga a que las Relaciones Internacionales como disciplina, para poder analizar los fenómenos globales, tengan que comprender los locales. En la actualidad es imposible $-\mathrm{o}$, en todo caso, contraproducente- concebir a nuestra disciplina exclusivamente desde lo internacional. Fenómenos como el Brexit no se pueden analizar en toda su complejidad si se omiten las particularidades locales del Reino Unido, su sistema político, la composición de la sociedad, el voto rural, etc. Tampoco se lo puede analizar solo desde lo interno sin indagar sobre el funcionamiento de la Unión Europea, las resistencias a la integración, el nuevo escenario internacional y el ascenso de los nacionalismos.

Especial énfasis quiero hacer sobre la Geopolítica. Los primeros estudios de Relaciones Internacionales estaban empapados de los aportes de esa disciplina. La Segunda Guerra Mundial y su asociación con el nazismo produjo, por un tiempo, una suerte de ocultamiento de esta disciplina como tal en los estudios internacionales. Pero, aunque sin nombrarla, el realismo y neorrealismo jamás dejaron de considerar sus aportes para los estudios internacionales. A partir de los años 60 y 70 volvió a formalizarse su inclusión. En nuestro país pasó algo similar en los años 80 y 90. La asociación de los estudios geopolíticos con la última dictadura militar hizo que este término fuera obviado o ignorado por mucho tiempo. Pero lo peor fue

\footnotetext{
${ }^{6}$ Véase Smouts, M. C. (1998). Les nouvelles relations internationales. Pratiques et theories. París: Presses de Sciences Po, p. 9. Disponible en: http://polcul.xoc.uam.mx/ polucl/pycls/marieclaudesmouts.pdf. (consulta: 20 de octubre del 2004).
} 
que no solo se ocultó el término, sino que los estudios geopolíticos disminuyeron y la mirada de los asuntos desde esta óptica tendió a desaparecer, lo que constituye una falencia grave en la proyección de un país.

Estos énfasis se evidencian en los planes de estudios de las carreras de licenciaturas en Relaciones Internacionales en el país. Un estudio realizado por Federico Merke (2005) que data del año 2005 pone de relieve que, en términos generales, el mayor énfasis está en lo que denomina el «núcleo duro» de la disciplina (p. 19), seguido de la Economía, la Ciencia Política, la Historia y el Derecho.

A la hora de analizar los perfiles de egresados, en las diecinueve universidades que brindan la carrera, destaca que en la mayoría de los casos hay un perfil enfocado en las Relaciones Internacionales y luego acompañado por la Economía, las ciencias jurídicas, la Ciencia Política y la Historia, con mayor o menor énfasis (Merke, 2005, pp. 21-23.).

Ahora bien, como el mismo autor afirma:

El problema central que se observa no reside en las orientaciones que cada universidad le da a la carrera. No hay nada negativo en ofrecer una buena cantidad de seminarios de historia o privilegiar la economía antes que el derecho. Son decisiones que toma cada universidad en función de sus intereses, sus valores y de los conocimientos disponibles entre sus profesores. E1 problema reside en la ausencia de un conjunto de materias que sean vistas como un núcleo duro que no puede ser soslayado. En este sentido, la discusión no pasa por discutir si se dicta más o menos historia, más o menos derecho sino por discutir la cantidad y calidad de los cursos de Relaciones Internacionales que deberían ser centrales a todas las carreras. (Merke, 2005, p. 23. Cursivas del original).
Un estudio más pormenorizado acerca de los programas de cada materia, la bibliografía seleccionada, el plantel docente, podría dar, eventualmente, otra perspectiva sobre los perfiles. También sería interesante indagar sobre el currículum oculto. En el trabajo de Merke se llama la atención acerca de la poca oferta sobre el tema de seguridad internacional $\mathrm{y}$ destaca los casos de la Universidad Católica de Salta, de la Universidad Católica de La Plata y del Instituto Universitario Policía Federal Argentina, que cuentan con materias como Estrategia, Geopolítica o Seguridad (2005, p. 21). ¿Por qué esa ausencia? La decisión de lo que se enseña implica, a la vez, una decisión acerca de lo que no se enseña, intencional o intencionalmente. No debe olvidarse que el currículum ejerce una función «reguladora» del contenido y que a la vez «(...) delimita los territorios de las asignaturas y especialidades» (Sacristán, 2010, p. 24).

\section{Camino a la transdisciplinariedad}

Ester Barbé destaca un pasaje de la obra de Quincy Wright en la que escribió que:

Las relaciones internacionales, en cuanto disciplina que contribuye a la comprensión, predicción, valoración y control de las relaciones entre los Estados y de las condiciones de la comunidad universal es, al mismo tiempo, una historia, una ciencia, una filosofía y un arte. (Quincy Wright en Barbé, 1995, p. 27).

Esta cita conduce al tema de la transdisciplinariedad de las Relaciones Internacionales. La transdisiplinariedad implica que las Relaciones Internaciones tienen una autonomía propia y un objeto de estudio propio y, desde su especificidad, atraviesan diversas disciplinas 
científicas de las cuales se sirven para poder realizar el abordaje de su objeto de estudio.

Es decir que las Relaciones Internacionales no son, como disciplina, meramente auxiliares de otras ciencias sociales; pero tampoco hay fundamentos para pretender hacer de las otras ciencias, disciplinas científicas, auxiliares de ella (Toledo, 2006, p. 64). Lo que sería mutuamente beneficioso es cumplir la función de disciplinas auxiliares unas de otras para una mejor comprensión de los fenómenos que estudian.

En ese sentido, en nuestro ámbito, las Relaciones Internacionales deberían atravesar a las otras disciplinas desde su especificidad, cuyo eje se vertebra - lo que no quiere decir que se agote - aún hoy en la Diplomacia.

A lo largo del tiempo se ha observado un avance hacia una concepción más flexible de la diplomacia y no solo por la irrupción de la paradiplomacia, que todavía genera ciertos debates terminológicos, sino porque otros agentes no públicos buscan impulsar la actividad diplomática desde la esfera de sus campos de acción.

Por citar, existen dos casos que están teniendo cada vez más predicamento. Alejo Antonio Jaime habla de la diplomacia de las organizaciones no gubernamentales (ONG), haciendo hincapié fundamentalmente en los grupos antiglobalización y los movimientos indigenistas (2019, pp. 241-258). Otro caso es la diplomacia científica, tecnológica y de la innovación, no como protesta sino como camino para la solución de problemas que aquejan al sistema internacional: medio ambiente, proliferación nuclear o seguridad cibernética, por ejemplo. Esto ya no es terreno exclusivo de lo académico, sino que hay áreas en las cancillerías de los países desarrollados que incorporan a la dimensión científica y la tecnológica, y hay informes de Gobierno, como el de España, sobre la diplomacia científica, y talleres e iniciativas a nivel internacional. ¿Qué pasa en los países periféricos como el nuestro? ¿Se está trabajando en estos temas? ¿Los planes de estudio de la carrera lo contemplan?

Otro tema, por ejemplo, la diplomacia del Twitter, o de las redes sociales. Esto ha puesto en perspectiva la necesidad de incorporar estudios de las comunicaciones sociales. Ciertamente, en el estudio las Relaciones Internacionales desde los años 50 del siglo XX se emplean teorías de las comunicaciones, pero no análisis de los medios de comunicación desde la óptica de las Relaciones Internacionales. ¿En dónde están estos temas en los estudios de la disciplina?

\section{Conclusiones}

A lo largo del artículo se ha intentado reflejar cómo el objeto de estudio de las Relaciones Internacionales y las discusiones y debates generados en torno a él incidieron decisivamente en la enseñanza de la disciplina. Se ha tratado de mostrar el hecho de que los contextos en los que surgió han sido fundamentales en su estructuración y, al mismo tiempo, que han conformado anclas que han sido difíciles de levantar para incluir nuevos contenidos acordes con la dinámica propia de las relaciones internacionales como actividad que despliegan los actores internacionales.

En el caso particular de la Argentina, tomando como eje el trabajo de Federico Merke, se observó que aún es débil la orientación hacia el núcleo duro de la disciplina, y que suele compartir espacio con otras disciplinas en los planes de estudios y en los perfiles de graduados pretendidos. Esta dependencia de miradas y enfoques incide inclusive en el tratamiento bibliográfico y en la edificación de sus contenidos, pues por lo general la estructuración de las carreras de Relaciones Internacionales muestra una fuerte impronta de las escuelas 
europeas, pero la bibliografía predominante en materia de teoría de las Relaciones Internacionales es estadounidense (Toledo, 2006, p. 16).

Muchos de los debates surgidos a raíz del estudio de las Relaciones Internacionales están arraigados en la concepción adoptada, ya sea como interdisciplinaria o multidisciplinaria. Ambas opciones conducen, en no pocas ocasiones, a desdibujar su esencia y a enfrascarse en interminables debates acerca de sus formalidades, metodologías, denominaciones y perspectivas, pero pocas veces en cuanto a sus contenidos. Resaltar su carácter transdisciplinario, en cambio, implica subrayar que hay una mirada específica de las Relaciones Internacionales que atraviesa a las otras disciplinas y que ese enfoque debe acompañar al dinamismo de los fenómenos que aborda.

Es necesario pensar en una flexibilización de los contenidos, en formas de estructuración más dinámicas y menos anquilosadas de nuestra disciplina. Constituye un desafío realmente grande y constituye una obligación acompañar esos cambios con planes de estudio y contenidos acordes.

\section{Referencias bibliográficas}

Barbé, E. (1995). Relaciones Internacionales. Madrid: Tecnos.

Beck, U. (1998). ¿Qué es la globalización? Falacias del globalismo, respuesta de la globalización. Buenos Aires: Paidós.

Carr, E. H. (2004). La crisis de los veinte años (1919-1939). Madrid: Catarata.

Del Arenal, C. (2000). Introducción a las Relaciones Internacionales. Madrid: Tecnos.
Dougherty, J.; Platzgraff, R. (1993). Teoría en pugna en las Relaciones Internacionales. Buenos Aires: G.E.L.

Jaime, A. (20109). «Activismo en México: Diplomacias de ONG en la política del siglo XXI». En Carrillo Velásquez, L. P. (coord.). El estudio de la acción social efectiva de las organizaciones. El caso de organizaciones de la sociedad civil en México. Ciudad de México: Universidad Nacional Autónoma de México, pp. 241-258.

Merke, F. (2005). «Relaciones Internacionales. El estado de la disciplina en la Argentina». Primer borrador de trabajo para ser presentado en las Terceras Jornadas de Investigación IDICSO-USAL, Buenos Aires, 14 de octubre. Recuperado de: http:// www.fts.uner.edu.ar/secretarias/academica/ rev_plan_estudio_cp/materiales_de_lectura/ ciencia_politica/Ciencia_Politica_en_Argentina/11_Merke_F-El_Estado_de_las_RRII en_la_ĀArgentina_2005.pdf [6-9-2020]

Sacristán, J. G. (2010). «¿Qué significa currículum?», en Sacristán, J. G. (comp.) Saberes e incertidumbres sobre el currículum. Madrid: Morata, pp. 21-44.

Smouts, M. C. (1998). Les nouvelles relations internationales. Pratiques et theories. París: Presses de Sciences Po. Recuperado de: http://polcul.xoc.uam.mx/ polucl/pycls/ marieclaudesmouts.pdf, p. 9. [Consulta: 20 de octubre de 2004].

Toledo, V. F. (2006). Introducción al estudio de las Relaciones Internacioanles. Salta: EUCASA.

Waltz, K. N. (1959). El hombre, el Estado y la guerra. Buenos Aires: Nova. 
